\title{
(2) OPEN ACCESS \\ Observational study of cytomegalovirus from breast milk and necrotising enterocolitis
}

\author{
Ravi Mangal Patel, ${ }^{1,2}$ Neeta Shenvi, ${ }^{3}$ Andrea Knezevic, ${ }^{3,4}$ Michael Hinkes, ${ }^{5}$ \\ George W Bugg, ${ }^{1,2}$ Sean R Stowell, ${ }^{6}$ John D Roback, ${ }^{6}$ Kirk A Easley, ${ }^{3}$ \\ Cassandra Josephson 1,2,6
}

\begin{abstract}
- Additional material is published online only. To view please visit the journal online (http://dx.doi.org/10.1136/ archdischild-2018-316613).
\end{abstract}

${ }^{1}$ Pediatrics, Emory University School of Medicine, Atlanta, Georgia, USA

${ }^{2}$ Pediatrics, Children's Healthcare of Atlanta, Atlanta, Georgia, USA

${ }^{3}$ Biostatistics and Bioinformatics, Emory University School of Public Health, Atlanta, Georgia, USA

${ }^{4}$ Epidemiology \& Biostatistics, Memorial Sloan-Kettering Cancer Center, New York, New York, USA

${ }^{5}$ Neonatology, Northside Hospital, Atlanta, Georgia, USA ${ }^{6}$ Pathology and Laboratory Medicine, Emory University School of Medicine, Atlanta, Georgia, USA

Correspondence to Dr Ravi Mangal Patel; rmpatel@emory.edu, ravipatelmd@gmail.com

Received 27 November 2018 Revised 19 June 2019 Accepted 25 June 2019
Check for updates

(C) Author(s) (or their employer(s)) 2020. Re-use permitted under CC BY-NC. No commercial re-use. See rights and permissions. Published by BMJ.

To cite: Patel RM, Shenvi N, Knezevic A, et al. Arch Dis Child Fetal Neonatal Ed 2020;105:F259-F265.

\section{ABSTRACT}

Objective To evaluate the relationship between cytomegalovirus (CMV) exposure from breast milk and risk of necrotising enterocolitis (NEC).

Design Secondary analysis of a multicentre, observational cohort study. Maternal breast milk and infant serum or urine were serially evaluated by nucleic acid testing at scheduled intervals for CMV. Infants with evidence of congenital infection were excluded. Competing-risks Cox models, with adjustment for confounders, were used to evaluate the relationship between breast milk CMV exposure or postnatal CMV infection and NEC.

Setting Three neonatal intensive care units in Atlanta, Georgia.

Patients Infants with a birth weight $\leq 1500$ grams. Exposures Maximal CMV viral load in breast milk in the first 14 days after birth or postnatal CMV infection. Two different approaches were used to assess the timing of onset of CMV infection (midpoint or early).

Main outcome measures NEC, defined as Bell stage II or greater.

Results Among 596 enrolled infants, 457 (77\%) were born to CMV seropositive mothers and 33 developed postnatal CMV infection (cumulative incidence $7.3 \%$, $95 \% \mathrm{Cl} 5.0 \%$ to $10.1 \%$ ). The incidence of NEC was $18 \%$ (6/33) among infants with CMV infection, compared with $7 \%$ (37/563) among infants without infection (adjusted cause-specific HR (CSHR): $2.81 ; 95 \% \mathrm{Cl} 0.73$ to 10.9 (midpoint); 6.02; 95\% Cl 1.28 to 28.4 (early)). Exposure to higher breast milk CMV viral load was associated with a higher risk of NEC (adjusted CSHR per twofold increase $1.28 ; 95 \% \mathrm{Cl} 1.06$ to 1.54 ).

Conclusions CMV exposure from breast milk may be associated with the development of NEC in very low birth weight infants.

\section{INTRODUCTION}

Cytomegalovirus (CMV) transmitted from blood transfusion or breast milk can cause postnatal infection in preterm infants. ${ }^{1}$ Prior studies, including from our group, have reported that maternal breast milk is the primary source of postnatal CMV infection among very low birth weight (VLBW) infants. ${ }^{12}$ In the USA, approximately 2800 premature infants develop breast milk-acquired postnatal CMV infection each year. ${ }^{3}$

Guidance in the US recommends that the benefits of human milk versus the risk of CMV transmission should be considered when deciding to breastfeed

\section{What is already known on this topic}

Breast feeding is beneficial for preterm infants, but many breastfed infants are exposed to cytomegalovirus (CMV) in maternal breast milk.

- There is conflicting data about the role of CMV as a cause of necrotising enterocolitis in very low birth weight infants.

\section{What this study adds}

- We found cytomegalovirus (CMV) infection and higher maternal breast milk (CMV) loads were associated with an increased risk of necrotising enterocolitis in breastfed infants.

- Our findings support the potential role of CMV from breast milk as a cause of necrotising enterocolitis.

VLBW infants born to mothers known to be CMVseropositive. ${ }^{4}$ Recent studies have demonstrated a potential association between postnatal CMV and necrotising enterocolitis (NEC) ${ }^{5} 6$ as well as other adverse outcomes including bronchopulmonary dysplasia (BPD). ${ }^{78}$ By contrast, other reports have found no association between postnatal CMV and NEC. ${ }^{910}$ Some prior studies have relied on clinically directed testing that could introduce diagnostic bias. Therefore, prospective cohort studies that include systematic testing for postnatal CMV are necessary to estimate the incidence of postnatal CMV and its relationship to neonatal outcomes. Our primary aim was to evaluate the association between breast milk CMV viral load, resultant postnatal CMV infection and the risk of NEC in VLBW infants. As a secondary aim, we evaluated the association between postnatal CMV infection and short-term adverse outcomes, including BPD, retinopathy of prematurity (ROP), sepsis or death.

\section{METHODS}

Study population

We conducted a secondary analysis of a multicentre observational birth-cohort study of transfusiontransmission of CMV in VLBW infants (TT-CMV study), with this study extending the initial cohort from 539 to 598 infants. ${ }^{11}$ We included infants born at three level III neonatal intensive care units in Atlanta, Georgia with follow-up from birth 
to 90 days, hospital discharge, transfer to a non-study affiliated hospital, or death. Inclusion criteria included: (1) birth weight $\leq 1500 \mathrm{~g}$ and (2) age $\leq 5$ days. Exclusion criteria included: (1) infant not expected to survive beyond 7 days of life; (2) severe congenital abnormality; (3) transfusion before enrolment; (4) maternal refusal to participate; or (5) congenital CMV infection. This study was approved by the Emory University Institutional Review Board and Research Oversight Committees at Grady Memorial Hospital and Northside Hospital and reported according to the Strengthening the Reporting of Observational Studies in Epidemiology statement. ${ }^{12}$

\section{CMV testing}

To determine maternal history of CMV infection, we tested maternal serum at study entry with a CMV IgG/IgM assay (online supplementary methods). ${ }^{1}$ For seronegative mothers, we performed CMV nucleic acid testing (NAT) on a maternal blood sample at study entry and conclusion to exclude infection that developed during the study. All infants underwent CMV NAT of residual blood samples or urine to ascertain CMV infection. Blood was tested using a sample after birth that was available at time of enrolment and at four time windows ( \pm 4 days) near days of life 21, 40, 60, 90 (or at discharge, if earlier); urine was collected as soon as possible after enrolment and at discharge if blood was not available. Breast milk samples were obtained from lactating mothers during weeks 1,3 and 4 , as well as days 34 to 40. Additional details on laboratory methods were previously described. ${ }^{1}$

\section{Definitions}

Congenital CMV infection was defined as a positive result of CMV NAT (or positive viral culture obtained from clinicianordered testing) in blood or urine samples obtained within 2 weeks of life. Postnatal CMV infection was defined as a positive CMV NAT or viral culture result in blood or urine after 2 weeks of life with a previously documented negative result. NEC was defined as Bell stage II or greater with adjudication of the staging of each NEC case as previously described. ${ }^{13}$ BPD was defined according to the consensus definition by the National Institutes of Health. ${ }^{14}$ ROP was defined as stage 2 or greater. Baseline illness severity was ascertained using the score for neonatal acute physiology. ${ }^{15}$ Race and ethnicity were determined by maternal self-report using options defined by federally funded study guidelines.

\section{Statistical analysis}

No sample size estimation was performed for this secondary analysis. The incidence of postnatal CMV infection was determined using the cumulative incidence function, as previously described. ${ }^{1}$ CIs were estimated using bootstrapping (1000 bootstrap samples), with the infant's mother as the clustering unit to account for multiple births. We compared baseline characteristics using one-way analysis of variance for normally distributed continuous variables, Kruskal-Wallis test for non-normally distributed continuous variables and $\mathrm{X}^{2}$ test for categorical variables, except in cases where expected cell counts were $<5$ where a Fisher's exact test was used. If overall group differences were significant, we performed pairwise comparisons between infants with and without postnatal CMV among those born to CMV seropositive mothers. We performed a competing risk analysis to estimate the cause-specific HR (CSHR) for NEC and mortality using a Cox proportional-hazards regression model using robust sandwich variance estimates to account for within-mother correlation that may occur in outcomes of multiple birth infants. ${ }^{16}$ Time-varying onset of infant postnatal CMV infection was estimated using two approaches (midpoint and earliest/ early) based on the postnatal age of the prior negative and subsequently positive NAT test and only NEC cases that occurred after the time-varying onset of infection were considered as events in the model (online supplementary figure 1). The assessment of $\log _{2}$ maximal breast milk CMV viral load was limited to the first 14 days of birth to avoid measuring the study exposure after the typical timing of onset of NEC. We also evaluated the two exposures of interest, both individually and together, to assess the independent contribution of CMV exposure in breast milk after accounting for whether or not an infant developed postnatal CMV infection. We adjusted for birth weight, race, premature rupture of membranes, maternal age and centre to address confounding, as these factors were significantly associated with either maternal CMV serostatus or the exposure or outcome of interest and reported both full and parsimonious models. For secondary outcomes, we used modified Poisson regression models with robust standard errors to estimate the relative risks between postnatal CMV infection and adverse short-term outcomes. ${ }^{17}$ To protect against model overfitting given the relatively low number of infants with postnatal CMV infection, models included only postnatal CMV, birth weight and centre. We defined statistical significance as a two-sided $\mathrm{p}$ value of less than 0.05 .

\section{RESULTS}

Of 600 VLBW infants enrolled in the cohort, 596 were evaluated for postnatal CMV infection (figure 1). All infants underwent NAT testing, with $98 \%$ of infants undergoing three or more assessments for CMV infection. Two infants $(0.3 \%)$ were diagnosed with congenital infection. The mean (SD) gestational age of the cohort was 27.9 (3.3) weeks (table 1). Overall, 296 $(49.5 \%)$ were female, 346 (57.9\%) were of Black race, and 520 $(87.0 \%)$ were fed at least some breast milk during the study. When comparing baseline characteristics among infants born to CMV seropositive mothers, including those with and without postnatal CMV infection, and those born to CMV seronegative mothers, we found no differences in gestational age, birth weight, sex, small for gestational age, or receipt of antenatal steroids among groups (table 1). However, infants born to CMV seropositive mothers were more commonly of Black race $(p<0.01)$. In addition, premature rupture of membranes was more common among CMV seropositive mothers and among infants with postnatal CMV infection.

\section{Incidence of postnatal CMV infection}

A total of 33 infants developed postnatal CMV infection (online supplementary table 1). The cumulative incidence of postnatal CMV at 12 weeks was 7.3\% (95\% CI 5.0\% to 10.1\%) overall and $9.7 \%$ (95\% CI $6.7 \%$ to $13.3 \%$ ) among infants born to CMV-seropositive mothers. The majority of CMV seropositive mothers had detectable CMV in breast milk $(76 \% ; 95 \% \mathrm{CI}$ $71 \%$ to $80 \%$ ), with $89 \%$ of mothers receiving breast milk testing. Approximately half of mothers had detectable CMV in breast milk in the first week of life with increases in viral load over the first 4-5 weeks after birth (online supplementary figure 2). However, we found no relationship between breast milk viral load and maternal IgG titre (online supplementary figure 3 and table 2). Among infants fed CMV positive breast milk, the cumulative incidence of postnatal $\mathrm{CMV}$ infection at 12 weeks was $16 \%$ (95\% CI $11 \%$ to $22 \%)$. 


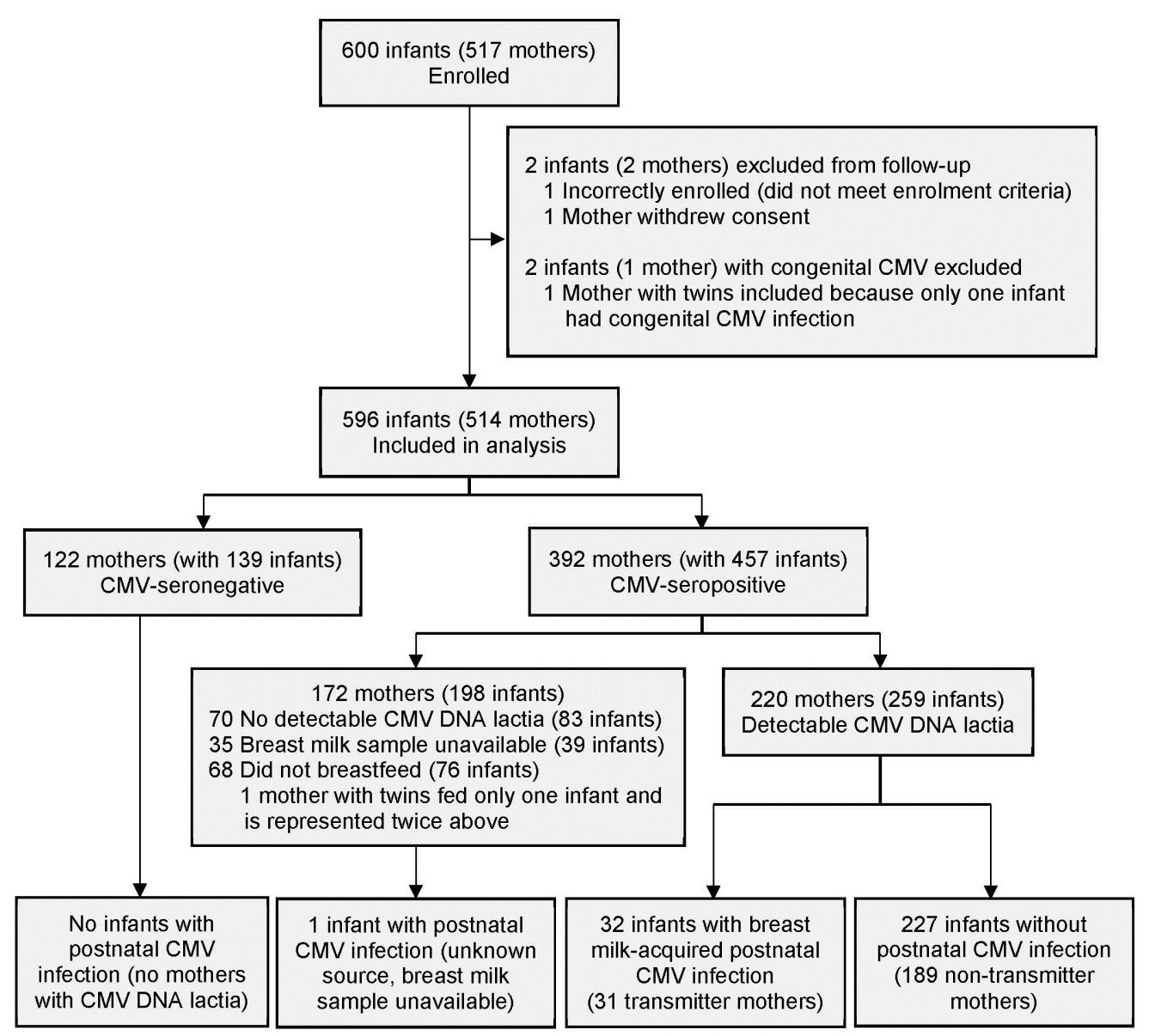

Figure 1 Study flow diagram. Numbers of infants and mothers enrolled and those subsequently excluded or included in analysis are shown by mother's CMV-serostatus, detectable CMV DNA lactia in maternal breast milk and whether the study infant developed postnatal CMV infection. Enrolled infants underwent follow-up from birth to 90 days, hospital discharge, transfer to a non-study affiliated hospital, or death. CMV, cytomegalovirus.

\section{Relationship between postnatal CMV infection and NEC}

The incidence of NEC was $18 \%$ (6/33) (8 week cumulative incidence: $15.2 \%, 95 \%$ CI $5.4 \%$ to $29.5 \%)$ among infants with postnatal CMV infection, compared with 7\% (37/563) (8 week cumulative incidence: $7.2 \%, 95 \% \mathrm{CI} 5.1 \%$ to $9.7 \%$ ) among infants without infection. Among infants with NEC $(n=43)$, $41 \%$ had occult blood in stool, $86 \%$ had pneumatosis, $26 \%$ had portal venous gas and $12 \%$ had pneumoperitoneum. Among the six NEC cases occurring in CMV positive infants, four were stage II (three with pneumatosis) and two were stage III (both with pneumoperitoneum) and all infants had negative initial CMV testing in blood or urine and had CMV positive maternal breast milk (online supplementary table 3). In multivariable analysis, postnatal CMV infection was associated with NEC using early estimates of onset (model B2), but the association diminished using a midpoint estimate of onset (model B1) (table 2). The maximal breast milk CMV viral load in the first 14 days of life was associated with NEC, with similar findings among enterally fed infants and those born to mothers with CMV positive breast milk. The mean maximal CMV viral load among mothers with infants who developed NEC $(n=20)$ was 19964 $\mathrm{IU} / \mathrm{mL}$ and among mothers whose infants did not develop NEC $(\mathrm{n}=118)$ was $5919 \mathrm{IU} / \mathrm{mL}$ (online supplementary table 4) In multivariable analyses, controlling for birth weight and postnatal CMV infection, the maximal breast milk CMV viral load in the first 14 days of birth was independently associated with NEC (CSHR per twofold increase or doubling: 1.26; 95\% CI 1.05 to
1.52) (table 2, model B3). Estimates of association were consistent in two expanded models, each including one of the two study exposures that also controlled for birth weight, maternal age, infant race, premature rupture of membranes and centre (table 2, models B4 and B5). In addition, we noted a certain dose response between the maximal viral load in breast milk in the first 14 days of life and risk of NEC, with viral load $>10016 \mathrm{IU} /$ $\mathrm{mL}$ associated with a 3.3 times higher risk (95\% CI 1.09 to 9.89) of NEC compared with infants with exposure to a CMV breast milk viral load of $\leq 1003 \mathrm{IU} / \mathrm{mL}$ (online supplementary table 5). There was no evidence that feeding only freeze-thawed breast milk, compared with fresh or freeze-thawed breast milk, was associated with a decreased risk of postnatal CMV infection or lower breast milk viral loads over time (online supplementary figures 4,5 ).

\section{Relationship between postnatal CMV infection and secondary outcomes}

The risks of death, BPD, ROP or a positive blood culture, were not significantly different among infants with and without postnatal CMV infection (table 3). There was no significant difference in the composite outcome of death, moderate-to-severe BPD, ROP Stage 3 or greater or NEC among infants with and without postnatal CMV (36\% vs 30\%; adjusted relative risk 1.14 ; $95 \%$ CI 0.77 to 1.69 ). 
Table 1 Baseline characteristics

\begin{tabular}{|c|c|c|c|c|c|}
\hline \multirow[b]{2}{*}{ Infant } & \multirow[b]{2}{*}{ Overall $n=596$} & \multirow[b]{2}{*}{$\begin{array}{l}\text { Mother CMV } \\
\text { seronegative } n=139\end{array}$} & \multicolumn{2}{|c|}{ Mother CMV seropositive } & \multirow[b]{2}{*}{ P value* $^{*}$} \\
\hline & & & $\begin{array}{l}\text { No postnatal CMV } \\
\text { infection } n=424\end{array}$ & $\begin{array}{l}\text { Postnatal CMV } \\
\text { Infection } n=33\end{array}$ & \\
\hline Gestational age-weeks, mean (SD) & $27.9(2.6)$ & $28.2(2.3)$ & $27.8(2.7)$ & $27.5(2.3)$ & 0.18 \\
\hline Birth weight- $-g$, mean (SD) & $1016(273)$ & $1036(269)$ & $1012(277)$ & $981(254)$ & 0.50 \\
\hline Male sex, n (\%) & $300(50)$ & $74(53)$ & $205(48)$ & $21(64)$ & 0.18 \\
\hline \multicolumn{6}{|l|}{ Race, n (\%) } \\
\hline Black & $345(58)$ & $50(36)$ & $277(65)$ & $18(55)$ & $<0.001$ \\
\hline White & $199(33)$ & $77(55)$ & $113(27)$ & $9(27)$ & \\
\hline Asian & $25(4.2)$ & $4(2.9)$ & $17(4.0)$ & $4(12)$ & \\
\hline More than one race & $23(3.9)$ & $7(5.0)$ & $14(3.3)$ & $2(6.1)$ & \\
\hline Othert & $4(0.7)$ & $1(0.7)$ & $3(0.7)$ & 0 & \\
\hline Hispanic ethnicity, n (\%) & $50(8.4)$ & $6(4.3)$ & $40(9.4)$ & $4(12)$ & 0.12 \\
\hline Singleton birth, $\mathrm{n}(\%)$ & $410(69)$ & $101(73)$ & $287(68)$ & $22(67)$ & 0.53 \\
\hline Small for gestational age, $\mathrm{n}(\%)$ & $105(26)$ & $31(31)$ & $68(24)$ & $6(27)$ & 0.38 \\
\hline Born outside study hospital, n (\%) & $7(1.2)$ & $2(1.4)$ & $5(1.2)$ & 0 & 0.79 \\
\hline 1 min Apgar score, $\neq$ median (IQR) & $5(4)$ & $5(5)$ & $5(5)$ & $5(4)$ & 0.03 \\
\hline 5 min Apgar score, $\neq$ median (IQR) & $8(2)$ & $9(1)$ & $8(2)$ & $7(2)$ & $<0.001$ \\
\hline Score for Neonatal Acute Physiology mean (SD) & $10.3(5.4)$ & $10.1(5.9)$ & $10.4(5.3)$ & $10.5(5.4)$ & 0.86 \\
\hline Mother & $n=514$ & $n=122$ & $n=360$ & $n=32$ & \\
\hline Age-years, mean (SD) & $29.5(6.5)$ & $29.4(5.9)$ & $29.4(6.7)$ & $30.7(7.2)$ & 0.55 \\
\hline At least one prenatal visit, $\mathrm{n}(\%)$ & $479(93.2)$ & $120(98.4)$ & $330(91.7)$ & $29(90.6)$ & 0.03 \\
\hline Premature rupture of membranes, $\S n(\%)$ & $195(38)$ & $44(36)$ & $129(36)$ & $22(69)$ & 0.001 \\
\hline Rupture of membranes>18 hours, $\S n(\%)$ & $109(21)$ & $29(24)$ & $68(19)$ & $12(38)$ & 0.04 \\
\hline Chorioamnionitis, n (\%) & 77 (15) & $10(8.2)$ & $63(18)$ & $4(13)$ & 0.04 \\
\hline Caesarean delivery, n (\%) & $387(75)$ & $96(79)$ & $268(74)$ & $23(72)$ & 0.58 \\
\hline Receipt of antenatal steroids, n (\%) & $429(83)$ & $104(85)$ & $298(83)$ & $27(84)$ & 0.81 \\
\hline \multicolumn{6}{|l|}{ Indications for premature delivery, $\mathrm{n}(\%)$} \\
\hline Isolated spontaneous labour & $150(29)$ & $27(22)$ & $117(33)$ & $6(19)$ & 0.04 \\
\hline Premature rupture of membranes & $141(27)$ & $35(29)$ & $89(25)$ & $17(53)$ & 0.002 \\
\hline Pregnancy-associated hypertension & $121(24)$ & $30(25)$ & $87(24)$ & $4(13)$ & 0.31 \\
\hline Fetal distress/Poor biophysical profile & $72(14)$ & $15(12)$ & $51(14)$ & $6(19)$ & 0.65 \\
\hline Bleeding complication & $51(9.9)$ & $12(9.8)$ & $36(10)$ & $3(9.4)$ & 0.99 \\
\hline
\end{tabular}

*Significant pairwise comparisons among infants with and without postnatal CMV infection as follows: premature rupture of membranes, $\mathrm{p}<0.001$; rupture of membranes $>18$ hours, $\mathrm{p}=0.01$; indication for delivery: premature rupture of membranes, $\mathrm{p}<0.001$.

tOther race category includes American Indian and Alaska Native, Native Hawaiian or Other Pacific Islander, or other unidentified race.

\$1 minute Apgar score is missing for four infants. $5 \mathrm{~min}$ Apgar score is missing for two infants.

§Rupture of membranes unknown for one mother.

CMV, cytomegalovirus.

\section{Characteristics of CMV testing and breast milk CMV expression among seropositive mothers}

Detection of postnatal CMV increased over postnatal age (online supplementary figure 6). The estimated incidence of postnatal CMV infection at 12 weeks that was diagnosed by blood NAT testing ( $\mathrm{n}=1969$ samples) was $6.0 \%$ (95\% CI $3.1 \%$ to $11.6 \%)$ and the incidence diagnosed by urine testing ( $\mathrm{n}=957$ samples) was $7.5 \%$ (95\% CI $3.7 \%$ to $15.3 \%$ ). CMV-seropositive mothers whose infants developed breast milk-acquired postnatal CMV infection had higher breast milk viral loads over the first 5 weeks of life (online supplementary table 6). In addition, higher maximal breast milk CMV viral load expression was associated with younger maternal age and first pregnancy, while there was no association with premature rupture of membranes, maternal race or receipt of antenatal steroids.

\section{DISCUSSION}

We found exposure to higher breast milk CMV viral load was associated with a higher risk of NEC in VLBW infants. Breast milk CMV viral load is the strongest risk factor for postnatal
CMV infection, ${ }^{1}$ which was also associated with NEC in our study. Our findings support the potential role of CMV as a cause of NEC. These findings are relevant to breastfeeding infants born to CMV seropositive mothers, because most CMV seropositive mothers will excrete CMV virus in their breast milk. ${ }^{12}$ Although breast milk feeding is well established as a protective factor against NEC, ${ }^{18}$ our findings could explain why some infants exclusively fed breast milk develop NEC.

Prior case reports have suggested a pathogenic role of CMV in NEC, ${ }^{6} 1920$ although systematic investigations have shown conflicting findings. In a study of tissue specimens from infants with surgical NEC, $81 \%$ of surgical NEC intestinal specimens were positive for CMV immediate early antigen, compared with $20 \%$ of control specimens $(\mathrm{p}=0.0002) .^{5}$ By contrast, a cohort study of 17 infants with NEC who underwent CMV nucleic acid testing by blood found no detectable virus in any of these infants. ${ }^{9}$ However, breastfeeding characteristics and the CMV sero-prevalence of the population were not reported to allow for comparisons to our cohort. Our findings of a significant association between breast milk viral load exposure, after controlling 
Original research

Table 2 Risk factors for NEC and mortality using competing-risks Cox regression models $(n=596)^{*}$

\begin{tabular}{|c|c|c|c|c|c|c|}
\hline \multirow[b]{2}{*}{ Risk factor } & \multirow[b]{2}{*}{ n (for NEC) } & \multirow[b]{2}{*}{$\mathbf{n}$} & \multicolumn{2}{|l|}{ NEC } & \multicolumn{2}{|l|}{ Mortality } \\
\hline & & & CSHR $(95 \% \mathrm{Cl})$ & $P$ value & CSHR $(95 \% \mathrm{Cl})$ & $P$ value \\
\hline \multicolumn{7}{|l|}{ A. Univariable analysis } \\
\hline Postnatal CMV—TDC (early)†‡ & 43 & 596 & 4.95 (1.08 to 22.6$)$ & 0.04 & 09 & \\
\hline Postnatal CMV—TDC (midpoint)†§ & 43 & 596 & $2.23(0.54$ to 9.20$)$ & 0.27 & 09 & \\
\hline Birth weight (per $100 \mathrm{~g}$ increase) & 43 & 596 & 0.75 (0.66 to 0.86$)$ & $<0.001$ & 0.52 (0.42 to 0.64 ) & $<0.001$ \\
\hline $\begin{array}{l}\text { Maximum CMV viral load in breast milk in first } 14 \text { days of } \\
\text { life (per twofold increase or doubling) }\end{array}$ & 43 & 596 & 1.37 (1.14 to 1.64$)$ & $<0.001$ & 0.81 (0.54 to 1.19 ) & 0.28 \\
\hline $\begin{array}{l}\text { Maximum CMV viral load in breast milk in first } 14 \text { days of } \\
\text { life, excluding infants never fed (per twofold increase or } \\
\text { doubling)** }\end{array}$ & 43 & 540 & 1.32 (1.09 to 1.59$)$ & 0.004 & 0.83 (0.56 to 1.23 ) & 0.34 \\
\hline $\begin{array}{l}\text { Maximum CMV viral load in breast milk in first } 14 \text { days of life } \\
\text { among infants fed CMV+breast milk (per twofold increase or } \\
\text { doubling)** }\end{array}$ & 26 & 259 & 1.35 (1.02 to 1.78$)$ & 0.03 & 0.85 (0.49 to 1.48$)$ & 0.57 \\
\hline Infant race (black vs non-black) & 43 & 596 & 1.72 (0.90 to 3.28$)$ & 0.10 & 1.01 (0.41 to 2.50$)$ & 0.99 \\
\hline Mother's age (per 1 year increase) & 43 & 596 & 0.98 (0.93 to 1.04$)$ & 0.50 & 1.02 (0.95 to 1.09 ) & 0.55 \\
\hline PROM (yes vs no) & 43 & 595 & 0.53 (0.27 to 1.07$)$ & 0.07 & 2.20 (0.89 to 5.43$)$ & 0.09 \\
\hline \multicolumn{7}{|l|}{ B1. Multivariable $(n=596)$} \\
\hline Postnatal CMV—TDC (midpoint)†§ & & & 2.81 (0.73 to 10.9$)$ & 0.13 & 09 & \\
\hline Birth weight (per $100 \mathrm{~g}$ increase) & & & 0.75 (0.65 to 0.86$)$ & $<0.001$ & 0.52 (0.41 to 0.65 ) & $<0.001$ \\
\hline \multicolumn{7}{|l|}{ B2. Multivariable $(n=596)$} \\
\hline Postnatal CMV—TDC (early)†‡ & & & 6.02 (1.28 to 28.4$)$ & 0.02 & 09 & \\
\hline Birth weight (per $100 \mathrm{~g}$ increase) & & & 0.75 (0.65 to 0.86$)$ & $<0.001$ & 0.52 (0.41 to 0.65 ) & $<0.001$ \\
\hline \multicolumn{7}{|l|}{ B3. Multivariable $(n=596)$} \\
\hline Postnatal CMV—TDC (early)† ‡ & & & 3.94 (0.77 to 20.0$)$ & 0.10 & 09 & \\
\hline Birth weight (per $100 \mathrm{~g}$ increase) & & & 0.76 (0.67 to 0.88$)$ & $<0.001$ & 0.49 (0.38 to 0.62 ) & $<0.001$ \\
\hline $\begin{array}{l}\text { Maximum CMV viral load in breast milk in first } 14 \text { days of } \\
\text { life (per twofold increase or doubling) }\end{array}$ & & & 1.26 (1.05 to 1.52$)$ & 0.02 & 0.71 (0.48 to 1.03$)$ & 0.07 \\
\hline \multicolumn{7}{|l|}{ B4. Multivariable $(n=596)$} \\
\hline Birth weight (per $100 \mathrm{~g}$ increase) & & & 0.77 (0.67 to 0.88$)$ & $<0.001$ & 0.47 (0.36 to 0.62$)$ & $<0.001$ \\
\hline $\begin{array}{l}\text { Maximum CMV viral load in breast milk in first } 14 \text { days of } \\
\text { life (per twofold increase ordoubling) }\end{array}$ & & & 1.28 (1.06 to 1.54$)$ & 0.01 & 0.73 (0.46 to 1.14$)$ & 0.17 \\
\hline Mother's age (per 1 year increase) & & & 1.00 (0.95 to 1.06$)$ & 0.78 & 1.00 (0.91 to 1.09 ) & 0.97 \\
\hline Infant race (black vs non-black) & & & 1.29 (0.64 to 2.57$)$ & 0.48 & 0.84 (0.34 to 2.12 ) & 0.72 \\
\hline PROM (yes vs no) & & & 0.55 (0.27 to 1.13$)$ & 0.10 & 2.26 (0.91 to 5.58$)$ & 0.08 \\
\hline \multicolumn{7}{|l|}{ B5. Multivariable $(n=596)$} \\
\hline Birth weight (per $100 \mathrm{~g}$ increase) & & & 0.75 (0.65 to 0.86$)$ & $<0.001$ & 0.49 (0.38 to 0.64$)$ & $<0.001$ \\
\hline Postnatal CMV—TDC (early)†‡ & & & 8.45 (1.83 to 38.9$)$ & 0.006 & 09 & \\
\hline Mother's age (per 1 year increase) & & & 1.00 (0.95 to 1.06$)$ & 0.93 & 1.02 (0.95 to 1.09$)$ & 0.65 \\
\hline Infant race (black vs non-black) & & & 1.34 (0.68 to 2.65$)$ & 0.40 & 0.86 (0.33 to 2.20$)$ & 0.74 \\
\hline PROM (yes vs no) & & & 0.48 (0.23 to 0.98$)$ & 0.04 & 2.49 (0.99 to 6.29$)$ & 0.05 \\
\hline
\end{tabular}

* Competing risks were 43 infants with NEC and 31 deaths total; 12 infants with NEC died and 19 deaths included in the mortality models. Two additional infants with congenital CMV infection were excluded (one infant diagnosed with NEC at age 15 days).

tPostnatal CMV infection included as a dichotomous time dependent covariate that can change, at most, once from uninfected to infected over 12 weeks of follow-up that is, once exposed always exposed.

$\ddagger$ Onset of postnatal CMV defined as time of earliest positive NAT result.

$\S$ Onset of postnatal CMV defined as the midpoint of last negative and first positive NAT result.

I) Of 19 non-NEC deaths, zero infants had postnatal CMV infection. Of note, 12 infants with NEC died.

**Fifty-six infants were not fed and were not included in the analysis.

Note: All multivariable models include adjustment for centre (estimates not shown). Doubling the maximum CMV viral load in breast milk in the first 14 days of life corresponds to an increment of 1 in the $\log _{2}$ scale.

CMV, cytomegalovirus; CSHR, cause specific HR; NAT, nucleic acid testing; NEC, necrotising enterocolitis; TDC, time dependent covariate; PROM, premature rupture of membranes.

for postnatal CMV detectable in an infant's blood or urine, suggests that a negative blood or urine test for CMV may not fully exclude the potential that breast milk CMV exposure is involved in the development of NEC. The mechanisms by which CMV causes NEC are unclear, but others have suggested that CMV may increase intestinal permeability and bacterial translocation, leading to exaggerated immune responses. ${ }^{21}$

We found no evidence of an association between postnatal CMV and risk of BPD, which contrasts recent studies reporting an association between the development of postnatal CMV and BPD. ${ }^{78}$ Differences between our findings and those of prior studies may be due to diagnostic bias, as we tested all enrolled infants for postnatal CMV while other studies evaluated only a small subset of infants who were symptomatic and underwent clinician-directed testing.

The overall estimated incidence of postnatal CMV infection in our study is similar to estimates from a prior systematicreview and meta-analysis in which the estimated incidence of 
Original research

Table 3 Association between postnatal CMV infection and secondary outcomes

\begin{tabular}{|c|c|c|c|c|}
\hline Outcome & Postnatal CMV+ & Postnatal CMV- & Relative risk* $(95 \% \mathrm{Cl})$ & $P$ value \\
\hline Death & $3 / 33(9.1 \%)$ & $28 / 563(5.0 \%)$ & $1.78(0.64,4.94)$ & 0.27 \\
\hline NEC & $6 / 33(18 \%)$ & $37 / 563(6.6 \%)$ & $\dagger$ & \\
\hline BPD (mild, moderate or severe) & $14 / 33(42 \%)$ & $226 / 563(40 \%)$ & 1.00 (0.69 to 1.46$)$ & 0.98 \\
\hline BPD (moderate or severe) & $8 / 33(24 \%)$ & $128 / 563(23 \%)$ & 1.02 (0.57 to 1.80$)$ & 0.95 \\
\hline ROP (stage 2+) & $4 / 33(12 \%)$ & $76 / 563(13 \%)$ & 0.85 (0.34 to 2.09$)$ & 0.72 \\
\hline ROP (stage 3+) & $1 / 33(3.0 \%)$ & $22 / 563(3.9 \%)$ & 0.73 (0.10 to 5.21$)$ & 0.75 \\
\hline Positive blood culture & $5 / 33(15 \%)$ & $59 / 563(10 \%)$ & 1.36 (0.68 to 2.74$)$ & 0.38 \\
\hline Composite outcome $\ddagger$ & $12 / 33(36 \%)$ & $171 / 563(30 \%)$ & $1.14(0.77$ to 1.69$)$ & 0.51 \\
\hline
\end{tabular}

*Results from modified Poisson regression models adjusted for birth weight and centre.

tPlease refer to table 2 for estimates of risk for NEC, accounting for temporality between exposure and outcome.

$\mp$ Defined as death, moderate or severe BPD, ROP stage 3+or NEC.

$\mathrm{BPD}$, bronchopulmonary dysplasia; CMV, cytomegalovirus; NEC, necrotising enterocolitis; ROP, retinopathy of prematurity.

breast milk-acquired postnatal CMV infection in VLBW infants was $6.5 \%(95 \%$ CI $3.7 \%$ to $10.9 \%)$ and the risk of postnatal CMV among infants fed untreated breast milk was $19 \%$ (11\%-32\%). Importantly, freezing breast milk did not prevent infection but was associated with slightly lower rates of postnatal CMV infection from breast feeding. Given the majority of CMV seropositive mothers will have CMV virus in their breast milk, evaluation of maternal serologic status could help identify those breastfed infants at risk for developing postnatal CMV infection, although universal CMV serologic screening is currently not recommended in the US. ${ }^{22}$ Risk stratification of infants could guide closer monitoring for postnatal CMV infection among those infants born to CMV seropositive mothers who are breastfed. Knowing maternal CMV serostatus could help determine if CMV testing could be informative for infants who develop NEC, although such testing would be less useful for infants who did not receive maternal breast milk or whose mothers were CMV seronegative. We found no relationship between maternal breast milk viral load and maternal IgG titre and it is unclear if neutralising antibodies can confer protection in prematurely born infants with limited trans-placental passage of antibodies. Potential strategies to reduce the risk of postnatal CMV include ultraviolet-C irradiation ${ }^{23}$ and pasteurisation of breast milk,,$^{24}$ and additional studies are needed to determine the optimal methods to safely decrease breast milk-acquired postnatal CMV infection.

The strengths of our study include the systematic and longitudinal assessment of CMV infection in our cohort, which allowed us to exclude infants with congenital infection and establish the temporality between the development of postnatal CMV or exposure to CMV positive breast milk and the subsequent development of NEC. In addition, we measured a number of potential confounders and controlled for these variables. Finally, we were able to measure CMV viral load in breast milk, the strongest predictor of development of postnatal CMV infection, and were able to quantify the independent risks associated with breast milk CMV virus exposure.

Our study has limitations. We potentially underestimated the risk of postnatal CMV by performing either serum or urine nucleic acid testing instead of only urine testing, although we found similar detection rates by either source and high concordance when both samples were tested. Although this is the largest cohort to systematically evaluate infants for postnatal CMV infection and NEC, our analysis of the relationship between postnatal CMV and short-term outcomes was not prespecified. Therefore, these posthoc analyses should be considered hypothesis-generating. In addition, we could not determine the exact age at onset of postnatal CMV infection as we did not test infants each day for CMV and therefore had to use two approaches (midpoint and early) that may not adequately reflect the temporal relationship between postnatal CMV infection and NEC. The limitation of this approach is suggested by the diminished estimates of association between postnatal CMV and NEC using the midpoint approach, which may be a more accurate estimate of onset of infection than the early approach. However, the more precisely estimated association between the amount of breast milk CMV virus exposure and NEC, in which more infants were exposed, suggests this may be a more common and important risk factor for NEC than postnatal CMV infection that is based on detection in an infant's blood or urine. We could not account for all potential confounders and additional studies are needed to better understand factors that lead to differing viral loads in maternal breast milk and the role of specific CMV strains. ${ }^{26}$ Finally, we did not evaluate intestinal tissue-specific findings of CMV and cases of NEC may have been reflective of CMV colitis. However, the majority of NEC cases among infants with CMV had pneumatosis or pneumoperitoneum, two wellestablished findings of NEC.

In conclusion, CMV exposure from breast milk may be associated with the development of NEC among breastfed VLBW infants. Additional studies are necessary to understand CMV transmission by breast milk and its consequences to guide prevention strategies given the known benefits of breast feeding in preterm infants.

Acknowledgements We thank all of the families who volunteered to participate in this study and the nurses, laboratory technologists, and physicians involved. We would like to acknowledge Tatiana Lanzieri, M.D., M.P.H. and Sheila Dollard, Ph.D. (Centers for Disease Control and Prevention in Atlanta, Georgia) for critical input and performing additional serologic testing on study samples, for which they did not receive financial compensation. Deborah AbdulAli, BS, MT(ASCP), Jessica Ingersoll, BS, MS, MT(ASCP), and Doris Igwe, BS, MLS(ASCP) (Emory University Department of Pathology), performed laboratory testing and Jane Skvarich, BSN, MN (Emory University, Department of Pathology), Katrina H. Grier, BSN (Northside Hospital), and Janna M. Benston, BSN (Northside Hospital), served as research nurses, for which they were provided salary support; Natia Saakadze (Emory University, Department of Pathology) performed serology testing and specimen processing, for which she was provided salary support. Angela M. Caliendo, MD, PhD (Alpert Medical School, Brown University, Providence, Rhode Island) provided critical input on CMV testing strategies. Becky Kinkead, PhD and the Children's Healthcare of Atlanta and Emory University Grant Editing \& Manuscript Support Core assisted in editing and formatting of the manuscript.

Contributors All authors agree to be accountable for all aspects of the work in ensuring that questions related to the accuracy or integrity of any part of the work are appropriately investigated and resolved. The following are the specific contributions of each study author: RMP designed the study, analysed and interpreted the data, drafted the initial version of the manuscript and approved the 
final manuscript as submitted. NS performed statistical analysis, interpreted the data, critically revised the manuscript and approved the final manuscript as submitted. AK performed statistical analysis, interpreted the data, critically revised the manuscript and approved the final manuscript as submitted. MH acquired study data, critically revised the manuscript and approved the final manuscript as submitted. GWB helped acquire study data, provided study supervision, critically revised the manuscript and approved the final manuscript as submitted. SRS helped interpret the data, revised the manuscript and approved the final manuscript as submitted. JDR designed the study, interpreted the data, provided study supervision, revised the manuscript and approved the final manuscript as submitted. KAE assisted in conceptualising and designing the study, supervised statistical analysis and helped interpret the data, critically revised the manuscript and approved the final manuscript as submitted. CJ obtained funding, designed the study, helped acquire data, analysed and interpreted the data, revised the manuscript and approved the final manuscript as submitted.

Funding This study was supported by the National Institutes of Health (NIH). RMP received salary support from the NIH National Center for Advancing Translational Sciences (NCATS) under awards UL1 TR000454 and KL2 TR000455 and from the National Heart Lung Blood Institute (NHLBI) under award K23 HL128942. The parent study was funded by the NHLBI under award P01 HL086773 (JDR and CJ).

Competing interests Dr. Patel received travel support from Danone Nutricia to attend the SIGNEC UK meeting.

Patient consent for publication Not required.

Provenance and peer review Not commissioned; externally peer reviewed.

Data sharing statement Data requestors should contact the corresponding author to request the relevant data and provide a protocol or outline for the proposed study that will use the data. After a data use agreement has been executed, the study data coordinating center will generate the requested data files, in a de-identified format, and provide appropriate accompanying documentation.

Open access This is an open access article distributed in accordance with the Creative Commons Attribution Non Commercial (CC BY-NC 4.0) license, which permits others to distribute, remix, adapt, build upon this work non-commercially, and license their derivative works on different terms, provided the original work is properly cited, appropriate credit is given, any changes made indicated, and the use is non-commercial. See: http://creativecommons.org/licenses/by-nc/4.0/.

\section{REFERENCES}

1 Josephson CD, Caliendo AM, Easley KA, et al. Blood transfusion and breast milk transmission of cytomegalovirus in very low-birth-weight infants: a prospective cohort study. JAMA Pediatr 2014;168:1054-62.

2 Hamprecht K, Maschmann J, Vochem M, et al. Epidemiology of transmission of cytomegalovirus from mother to preterm infant by breastfeeding. Lancet 2001;357:513-8.

3 Lanzieri TM, Dollard SC, Josephson CD, et al. Breast milk-acquired cytomegalovirus infection and disease in VLBW and premature infants. Pediatrics 2013;131:e1937-45.

4 Centers for Disease Control and Prevention. Cytomegalovirus (CMV) and Congenital CMV Infection. 2016 https://www.cdc.gov/cmv/clinical/features.html (accessed 10 Jan 2017).

5 Omarsdottir S, Agnarsdottir M, Casper C, et al. High prevalence of cytomegalovirus infection in surgical intestinal specimens from infants with necrotizing enterocolitis and spontaneous intestinal perforation: A retrospective observational study. J Clin Virol 2017:93:57-64.
6 Tengsupakul S, Birge ND, Bendel CM, et al. Asymptomatic DNAemia heralds CMV-associated NEC: case report, review, and rationale for preemption. Pediatrics 2013;132:e1428-34.

7 Mukhopadhyay S, Meyer SA, Permar SR, et al. Symptomatic Postnatal Cytomegalovirus Testing among Very Low-Birth-Weight Infants: Indications and Outcomes. Am J Perinatol 2016;33:894-902.

8 Kelly MS, Benjamin DK, Puopolo KM, et al. Postnatal Cytomegalovirus Infection and the Risk for Bronchopulmonary Dysplasia. JAMA Pediatr 2015;169:e153785.

9 Skeath T, Stewart C, Waugh S, et al. Cytomegalovirus and other common enteric viruses are not commonly associated with NEC. Acta Paediatr 2016;105:50-2.

10 Turner KM, Lee HC, Boppana SB, et al. Incidence and impact of CMV infection in very low birth weight infants. Pediatrics 2014;133:e609-15.

11 Josephson CD, Castillejo MI, Caliendo AM, et al. Prevention of transfusion-transmitted cytomegalovirus in low-birth weight infants $(\leq 1500 \mathrm{~g})$ using cytomegalovirusseronegative and leukoreduced transfusions. Transfus Med Rev 2011;25:125-32.

12 von Elm E, Altman DG, Egger M, et al. Strengthening the Reporting of Observational Studies in Epidemiology (STROBE) statement: guidelines for reporting observational studies. BMJ 2007;335:806-8.

13 Patel RM, Knezevic A, Shenvi N, et al. Association of Red Blood Cell Transfusion, Anemia, and Necrotizing Enterocolitis in Very Low-Birth-Weight Infants. JAMA 2016;315:889-97.

14 Jobe AH, Bancalari E. Bronchopulmonary dysplasia. Am J Respir Crit Care Med 2001;163:1723-9.

15 Richardson DK, Gray JE, McCormick MC, et al. Score for Neonatal Acute Physiology: a physiologic severity index for neonatal intensive care. Pediatrics 1993;91:617-23.

16 Austin PC, Lee DS, Fine JP. Introduction to the analysis of survival data in the presence of competing risks. Circulation 2016;133:601-9.

17 Zou G. A modified poisson regression approach to prospective studies with binary data. Am J Epidemiol 2004;159:702-6.

18 Meinzen-Derr J, Poindexter B, Wrage L, et al. Role of human milk in extremely low birth weight infants' risk of necrotizing enterocolitis or death. J Perinatol 2009;29:57-62.

19 Bar-Meir M, Farrow KN, Melin-Aldana H, et al. Cytomegalovirus Enterocolitis Mimicking Necrotizing Enterocolitis: Case Reports and Review of the Literature. $J$ Pediatric Infect Dis Soc 2013;2:71-5.

20 Goelz R, Hamprecht K, Klingel K, et al. Intestinal manifestations of postnatal and congenital cytomegalovirus infection in term and preterm infants. J Clin Virol 2016;83:29-36.

21 Tran L, Ferris M, Norori J, et al. Necrotizing enterocolitis and cytomegalovirus infection in a premature infant. Pediatrics 2013;131:e318-22.

22 American College of Obstetricians and Gynecologists. Practice bulletin no. 151: Cytomegalovirus, parvovirus B19, varicella zoster, and toxoplasmosis in pregnancy. Obstet Gynecol 2015;125:1510-25.

23 Lloyd ML, Hod N, Jayaraman J, et al. Inactivation of Cytomegalovirus in Breast Milk Using Ultraviolet-C Irradiation: Opportunities for a New Treatment Option in Breast Milk Banking. PLoS One 2016;11:e0161116.

24 Hamprecht K, Maschmann J, Müller D, et al. Cytomegalovirus (CMV) inactivation in breast milk: reassessment of pasteurization and freeze-thawing. Pediatr Res 2004;56:529-35.

25 Stock K, Griesmaier E, Brunner B, et al. Pasteurization of breastmilk decreases the rate of postnatally acquired cytomegalovirus infections, but shows a nonsignificant trend to an increased rate of necrotizing enterocolitis in very preterm infants--a preliminary study. Breastfeed Med 2015;10:113-7.

26 Novak Z, Ross SA, Patro RK, et al. Cytomegalovirus strain diversity in seropositive women. J Clin Microbiol 2008:46:882-6. 